\title{
Perseveración de respuesta y psicopatía subclínica*
}

\author{
PABLO RIBES GUARDIOLA \\ pribes@uji.es \\ ROSARIO POY GIL \\ poy@uji.es \\ RAÚl LÓPEZ PENAdÉS \\ penades@uji.es \\ Pilar Segarra Cabedo \\ segarra@uji.es \\ ÀngELS ESTELLER CANO \\ aestelle@uji.es \\ Alicia Fonfría MORENO \\ afonfria@uji.es \\ CARLOS VENTURA BORT \\ cventura@uji.es \\ JAVIER MOLTÓ BROTONS \\ molto@uji.es
}

\section{Resumen}

Una de las características más salientes de la psicopatía es la incapacidad para aprender de la experiencia (Cleckley, 1941/1976), lo que define a los psicópatas como individuos con dificultades para anticipar las consecuencias adversas de sus acciones y propensos a perseverar en conductas desadaptativas. Estudios previos mediante la Card Perseveration Task (CPT; Newman et al., 1987) revelan la existencia de una perseveración de respuestas no adaptativas (mayor número de jugadas y menores ganancias) en psicópatas. Además, este déficit parece estar relacionado con las características de desviación social e impulsividad del trastorno y con una falta de reflexión tras el feedback de recompensa y de castigo (Moltó et al., 2007).

Con el objetivo de extender y replicar este déficit en poblaciones no penitenciarias, se administró la CPT a una muestra de 80 universitarios varones evaluados mediante el Psychopathic Personality Inventory-Revised (PPI-R; Lilienfeld y Widows, 2005).

No se encontró evidencia de una perseveración de respuestas asociada a las puntuaciones totales ni por factores del PPI-R. Solo la subescala de 'Ausencia Despreocupada

\footnotetext{
* Esta investigación ha sido posible gracias a la financiación de los proyectos SEJ2007-61742 del Ministerio de Ciencia e Innovación y PSI2011-22559 del Ministerio de Economía y Competitividad.

Correspondencia: Javier Moltó, Laboratorio de Neurociencia Afectiva, Departamento de Psicología Básica, Clínica y Psicobiología, Universitat Jaume I, Avenida Sos Baynat s/n, 12071, Castellón, España. E-mail: molto@uji.es.
} 
de Planes' se relacionó con un mayor número de cartas jugadas, menores ganancias y menores tiempos de reflexión tras castigo y tras recompensa $(11.6 \%, 8.1 \%, 10.8 \%$ y $6.8 \%$ de la varianza explicada, respectivamente).

Estos resultados constituyen un soporte empírico a la escala de 'Ausencia Despreocupada de Planes' como medida de las características de impulsividad, falta de planificación y dificultades para aprender de la experiencia. Asimismo, establecen cierto grado de continuidad entre la población normal y la penitenciaria en la perseveración de respuestas no adaptativas y la falta de reflexión general asociada a las características de impulsividad de la psicopatía.

Pablabras clave: psicopatía subclínica, perseveración de respuestas, Psychopathic Personality Inventory-Revised (PPI-R), Card Perseveration Task (CPT), ausencia despreocupada de planes.

\section{Abstract}

One of the most salient characteristic of psychopathy is the failure to learn from the experience (cleckley, 1941/1976), thus defining psychopaths as individuals with difficulties to anticipate the adverse consequences of their acts, and prone to perseverate in their maladaptative responses. Previous studies using the Card Perseveration Task (CPT; Newman et al., 1987) have showed evidence of maladaptative response perseveration -more cards played and less money earned- in incarcerated psychopaths. Furthermore, this deficit seems to be related to the social deviance and impulsivity features of the disorder and to a lack of reflection after reward and punishment feedback (Moltó et al., 2007).

In order to extend and replicate this deficit in nonincarcerated samples, we administered the CPT to a sample of 80 male undergraduates assessed for psychopathic traits with the Psychopathic Personality Inventory-Revised (PPI-R; Lilienfeld \& Widows, 2005).

No evidence of maladaptative response perseveration associated with PPI-R total and factor scores was found. However, 'Carefree Nonplanfulness' scale scores were related to more cards played, less money earned, and shorter response times after reward and punishment feedback $(11.6 \%, 8,1 \%, 10,8 \%$ and $6,8 \%$ of the variance, respectively. These results provide empirical support to the 'Carefree Nonplanfulness' scale as a measure of impulsivity, lack of forethought and failure to learn from experience. Additionally, our results suggest some degree of continuity of maladaptative response perseveration and lack of overall reflection associated with the impulsivity features of psychopathy in community and incarcerated populations.

Keywords: Subclinical Psychopathy, Response Perseveration, Psychopathic Personality Inventory-Revised (PPI-R), Card Perseveration Task (CPT), Carefree Nonplanfulness. 


\section{Introducción}

La psicopatía es un trastorno de la personalidad en el que confluyen tanto rasgos de carácter afectivo/interpersonal (crueldad, manipulación, egocentrismo, falta de empatía, frialdad emocional, ausencia de miedo, etc.) como de desviación social (irresponsabilidad, impulsividad, agresividad, etc.; Cleckley, 1941/1976; Hare, 1991).

Una de las características más salientes de este trastorno es la incapacidad para aprender de la experiencia, lo que define a los psicópatas como individuos con dificultades para anticipar las consecuencias adversas de sus acciones (Cleckley, (1941/1976). De esta forma, su conducta es, a menudo, antisocial y es probable que persista en el futuro.

La evidencia empírica sugiere que los psicópatas presentan déficits a la hora de inhibir respuestas que previamente han sigo castigadas en situaciones de competición entre señales de recompensa y castigo (Newman, Patterson y Kosson, 1987; Siegel, 1978). En este sentido, se ha sugerido que el mecanismo psicológico que subyace a este tipo de déficit es una deficiente modulación de respuestas (Patterson y Newman, 1993; Newman y Wallace, 1993). Así, la conducta desinhibida de los psicópatas y sus dificultades para evitar castigos y aprender de la experiencia podría estar causada por dificultades para modificar o detener una tendencia de respuesta dominante hacia una recompensa y procesar las señales de castigo (o el feedback negativo sobre su ejecución) que indican que esa conducta debe ser interrumpida. De esta forma, los psicópatas son propensos a perseverar en sus respuestas desadaptativas, quedando definido este proceso como el mantenimiento de una tendencia de respuesta hacia la recompensa a pesar del castigo o de los cambios en las contingencias ambientales que hacen que la respuesta continuada no resulte adaptativa (McCleary, 1966).

La existencia de una perseveración de respuesta desadaptativa en la psicopatía y en otros grupos psicopatológicos ha sido probada en el laboratorio mediante la Tarea de Perseveración de Cartas (Card Perseveration Task, CPT; Newman et al.,1987). La CPT consiste en un mazo computerizado de 100 cartas, ordenado de tal forma que la probabilidad inicial de obtener una recompensa (ganar dinero) va decreciendo de manera lineal a medida que avanza el juego. Así, existe una tasa inicial del $90 \%$ de recompensa (y $10 \%$ de castigo) en las 10 primeras jugadas, que va decreciendo linealmente un $10 \%$ en cada bloque de 10 cartas jugadas, hasta que en las 10 últimas jugadas existe una probabilidad del $0 \%$ de obtener una recompensa y del $100 \%$ de obtener un castigo en cada jugada. Esta tarea evalúa la perseveración de respuestas en tanto que permite establecer en las primeras jugadas una clara tendencia de respuesta hacia la recompensa, y evalúa la habilidad de los sujetos para modificar esa tendencia de respuesta inicial hacia la recompensa de acuerdo con los cambios en las contingencias ambientales que van convirtiendo dicha tendencia inicialmente adaptativa (recompensada) en una tendencia desadaptativa (castigada).

Mediante esta tarea se ha podido poner de manifiesto la existencia de una perseveración de respuestas no adaptativas en muestras de psicópatas encarcelados varones (Newman et al., 1987; Moltó, Poy, Segarra, Pastor y Montañés, 2007) -aunque no en muestras de mujeres institucionalizadas (Vitale y Newman, 2001). Así, mediante la CPT, Newman et al. (1987) demostraron que en los psicópatas se daba una perseveración de respuestas desadaptativa -jugaron más cartas y obtuvieron menos ganancias- en comparación con los no psicópatas cuando jugaban libremente. Sin embargo, cuando tras cada jugada se imponía una pausa de 5 segundos, no se encontraron diferencias entre ambos grupos en el número de cartas jugadas ni en las ganancias obtenidas. Los autores concluyeron que la pausa impuesta tras el resultado de cada jugada permitía a los psicópatas utilizar mejor el feedback sobre su respuesta, posibilitando así un mejor procesamiento de la información sobre los cambios en la probabilidad de ser castigados y, por tanto, reduciendo su perseveración desadaptativa. Sin 
embargo, el papel que juega la reflexión tras el feedback en el déficit de perseveración no fue examinada experimentalmente. Esta cuestión fue puesta a prueba por Moltó et al. (2007) en una muestra penitenciaria evaluada mediante el Psychopathy Checklist-Revised (PCL-R; Hare, $1991,2003)$ a la que administraron una adaptación de la CPT en la que se registraba la latencia de respuesta tras el feedback. Los resultados de este estudio replicaron los obtenidos por Newman et al. (1987): los psicópatas jugaron más cartas y ganaron menos dinero que los grupos mixto y de no psicópatas. Mediante el análisis de la latencia de respuesta tras el feedback demostraron asimismo que la perseveración de respuestas no adaptativas de los psicópatas se debía, más que a una falta de reflexión tras el feedback negativo, a una falta de reflexión general, ya que tanto los tiempos de reacción tras castigo como tras recompensa correlacionaban de manera inversa con el número de cartas jugadas y directa con las ganancias totales en la tarea.

Por otra parte, este estudio examinó el papel de los diversos componentes del trastorno en la ejecución de la CPT. Así, los análisis de regresión jerárquica múltiple mostraron que las características de desviación social del trastorno (Factor 2 del PCL-R), en particular el estilo de vida impulsivo e irresponsable (Faceta 3 del PCL-R; véase Hare, 2003), predecían de manera única la peor ejecución en la tarea por parte de los psicópatas. Las puntuaciones en el Factor 2 y en la Faceta 3 del PCL-R se asociaban, además, con menores tiempos de reflexión tras recompensa y tras castigo. La importancia de la falta de reflexión general en la perseveración de respuestas no adaptativas, junto con la clara asociación entre las características de desviación social de la psicopatía y la dimensión externalizante de la personalidad y la psicopatología ( $r=.84$; Patrick, Hicks, Krueger y Lang, 2005), sugieren que la perseveración de respuestas encontrada en psicópatas no constituye un déficit específico del trastorno. Más bien parece definir, de forma más general, la conducta de otros grupos psicopatológicos relacionados con el factor general de vulnerabilidad externalizante (Krueger, Hicks, Patrick, Carlson, lacono y McGue, 2002; Krueger, Markon, Patrick, Benning y Kramer, 2007), caracterizados por la conducta impulsiva, la agresividad, la falta de reflexión y la persistencia en conductas apetitivas a pesar de las consecuencias adversas.

En línea con esta idea, varios estudios han demostrado la existencia de perseveración de respuestas no adaptativas en diversos grupos psicopatológicos estrechamente relacionados con la dimensión externalizante de la personalidad y la psicopatología. Así, se ha encontrado evidencia de perseveración de respuestas no adaptativas en grupos de delincuentes juveniles (Fonseca y Yule, 1995; White, Moffit, Caspi, Bartusch, Needles y Stouithamer-Loeber, 1994), en niños y adolescentes en escuelas de educación especial por problemas emocionales y dificultades conductuales, con tendencias psicopáticas (Fisher y Blair, 1998) o con características de trastorno disocial (Shapiro, Quay, Hogan y Schwartz, 1988), en niños con diagnóstico de trastorno disocial (Fonseca y Yule, 1995), en niños con trastorno disocial con o sin déficit de atención e hiperactividad (TDAH) (Matthys, Van Goozen, De Vries, Cohen-Kettenis y Van Engeland, 1998), en adultos jóvenes con trastorno disocial e historia de trastorno por hiperactividad (Fischer, Barkley, Smallish y Fletcher, 2005), en niños con trastorno negativista desafiante (Matthys, Van Goozen, Snoek y Van Engeland, 2004), en niños agresivos (Séguin, Arseneault, Boulerice, Harden y Tremblay, 2002), y en jugadores patológicos (Goudriaan, Oosterlaan, De Beurs y Van den Brink, 2005). Además, la peor ejecución en la CPT correlaciona con síntomas de dependencia de diferentes drogas (Martin, Rayens, Kelly, Hartung y Leukefeld, 2000).

El hecho de que las relaciones observadas entre la psicopatía y la perseveración de respuestas no adaptativas se expliquen específicamente a través de las características de desviación social del trastorno (Moltó et al., 2007) respaldan la conceptuación de la psicopatía como un constructo multifacético (Skeem, Polaschek, Patrick y Lilienfeld, 2011). Recien- 
tes modelos etiológicos han propuesto que las distintas manifestaciones fenotípicas de la psicopatía -esto es, las características afectivo-interpersonales del trastorno, por un lado, y las características de desviación social, por otro- son consecuencia de distintos procesos etiológicos relativamente independientes (Fowles y Dindo, 2006, 2009; Patrick, 2007; Patrick \& Bernat, 2009). Desde esta perspectiva, una disposición temperamental de bajo miedo (que teóricamente refleja una hiporreactividad del sistema motivacional defensivo) subyacería a parte de las características de tipo afectivo/interpersonal del trastorno, mientras que el concepto disposicional de vulnerabilidad externalizante (que refleja deterioros en los sistemas frontales que median la anticipación, la planificación y el control conductual) estaría relacionado con las manifestaciones de desviación social del trastorno (Patrick y Bernat, 2009).

En línea de lo anteriormente expuesto, el objetivo del presente trabajo es examinar si existe una perseveración de respuestas no adaptativas en una muestra subclínica (o no institucionalizada), evaluada mediante el Psychopathic Personality Inventory-Revised (PPI-R; Lilienfeld y Widows, 2005). Dicho instrumento ha emergido en los últimos años como una herramienta prometedora para el estudio de la psicopatía en muestras no penitenciarias, ya que fue desarrollado para evaluar las características de personalidad y rasgos disposicionales asociados a la psicopatía basados en las descripciones prototípicas avanzadas por Cleckley, sin hacer referencia explícita a la conducta criminal o antisocial.

Teniendo en cuenta la evidencia empírica revisada anteriormente, en el presente estudio esperamos encontrar evidencia de (1) una perseveración de respuestas no adaptativas (mayor número de cartas jugadas y menores ganancias) asociada con las características de impulsividad de la psicopatía, y (2) que esta perseveración de respuestas no adaptativas se relaciona con una falta de reflexión general (menores tiempos de reacción tras el feedback de recompensa y castigo) y no exclusivamente con una falta de reflexión tras el feedback negativo (cf. Moltó et al., 2007).

\section{Método}

\section{Participantes}

La muestra experimental estaba compuesta por 80 estudiantes universitarios varones de la Universitat Jaume I de Castellón que participaban en un estudio más amplio sobre personalidad y psicopatía, con edades comprendidas entre los 17 y los 45 años ( $M=21.45$ ). Para su evaluación se utilizó la adaptación española del Psychopathic Personality Inventory-Revised (PPI-R; Lilienfeld y Widows, 2005) (véase López, 2010). El PPI-R es un autoinforme que consta de 154 ítems que evalúan los rasgos de personalidad nucleares del trastorno. Los ítems se puntúan en una escala tipo Likert de cuatro puntos $(1=$ falso, 2 = más falso que verdadero, 3 = más verdadero que falso, 4 = verdadero). Este cuestionario proporciona una puntuación global de psicopatía, resultado de la suma directa de las puntuaciones en cada una de las 8 subescalas de contenido que lo conforman. Diversos estudios factoriales exploratorios del PPI (Benning, Patrick, Hicks, Blonigen y Krueger , 2003; Lilienfeld y Widows, 2005) han mostrado que 7 de las 8 subescalas de contenido se pueden agrupar en dos factores generales. El primer factor, PPI-I O «Dominancia/Ausencia de miedo», está compuesto por las subescalas de Ausencia de Miedo, Inmunidad al Estrés e Influencia Social, y parece reflejar las características de tipo afectivo/interpersonal de la psicopatía. El segundo factor, PPI-II o «lmpulsividad Egocéntrica», agrupa las escalas de Egocentrismo Maquiavélico, Inconformismo Rebelde, Ausencia Despreocupada de Planes y Externalización de la Culpa, y parece reflejar las características de desviación social y externalizantes del trastorno como la impulsividad, la 
agresividad y el no cumplimiento de las normas sociales. La escala de «Frialdad Emocional» no satura en ningún factor.

La muestra experimental definitiva, una vez excluidos los participantes con un perfil no válido en el PPI-R $(n=6)$, con menos de 10 jugadas en la CPT (i. e., sin una tendencia dominante de respuesta hacia la recompensa, o sin tiempos de reflexión tras castigo; $n=7$ ), o con tiempos de reflexión significativamente dispares de la media de la muestra experimental (técnica Jacknife; $n=5)$, fue de 62 participantes.

\section{Procedimiento}

Previamente a la sesión de laboratorio, se administró a un grupo más amplio de participantes una batería de autoinformes de psicopatía y personalidad. Los participantes recibían por su colaboración en la sesión de cuestionarios puntos extra en las asignaturas de los profesores responsables del laboratorio. Posteriormente, se contactó por teléfono con la muestra final de 80 sujetos para acudir a la sesión experimental en el laboratorio, en la que, además de la CPT, se realizaron otras tareas experimentales que serán expuestas en otros trabajos.

Una vez en el laboratorio, a los participantes se les administró una adaptación de la tarea de perseveración de cartas de Newman et al. (1987), similar a la utilizada por Moltó et al. (2007), en la que se registraba la latencia de respuesta tras el feedback. La presentación de la tarea y la adquisición de los datos fue controlada a través de un ordenador PC HP workstation xw4600 con un Monitor 22» LCD TFT, mediante la aplicación Presentation v.9.2 (Neurobehavioral Systems). Las respuestas de los participantes fueron registradas mediante el plafón de respuesta $\mathrm{RB}-730$ de Cedrus.

Al comienzo de la tarea, en la pantalla del ordenador los participantes veían el dorso de una carta de la baraja española y dos montones de 50 monedas de 10 céntimos de euro. El montón de monedas de la izquierda era el del sujeto experimental y se le informaba de que 20 de las monedas le pertenecían por participar y las otras 30 se le prestaban para empezar el juego. El montón de la derecha representaba la cantidad de monedas que el sujeto podía ganar. Cada ensayo se iniciaba con la pregunta «¿Quieres jugar?» encima del dorso de la carta de la baraja. Los participantes entonces decidían entre dos opciones: «jugar» (pulsando el botón derecho del plafón de respuesta) o bien «dejar de jugar» (pulsando el botón izquierdo del plafón se respuesta), siempre y cuando se hubiera jugado al menos la primera vez. Tras cada jugada, desaparecía la pregunta y la carta se daba la vuelta. Si la carta era una figura (sota, caballo, rey o as), aparecía la palabra «¡GANAS!» durante $1000 \mathrm{~ms}$, sonaba un tono agudo y se añadía una moneda de 10 céntimos de euro al montón del sujeto. Si la carta era un número (del 2 al 7) aparecía la palabra «jPIERDES!», sonaba un zumbido, y se perdía una de las monedas de 10 céntimos del montón del sujeto.

La tarea estaba compuesta por 100 cartas de la baraja española que se presentaban en un orden predefinido, de manera que en las 10 primeras jugadas existía una probabilidad de 0.9 de obtener una recompensa (ganar 10 céntimos de euro) y 0.1 de obtener un castigo (perder 10 céntimos). Dicha probabilidad se reducía de manera lineal un 0.1 en cada bloque de 10 cartas jugadas, hasta que en las últimas 10 jugadas la probabilidad de obtener una recompensa era de 0.

Los participantes se sentaban en una habitación insonorizada y con baja iluminación. El experimentador acompañaba a los sujetos a la habitación y leía en voz alta las instrucciones de la tarea que aparecían en pantalla. 
Las variables dependientes estudiadas fueron el número de cartas jugadas, las ganancias totales obtenidas y los tiempos de reflexión tras castigo y tras recompensa (en ms).

Con el objetivo de examinar la relación entre las puntuaciones en el PPI-R y la perseveración de respuestas no adaptativas en la psicopatía subclínica, se llevaron a cabo 3 conjuntos de modelos lineales generales (MLG) sobre cada variable dependiente (número de jugadas, ganancias, tiempo de reflexión tras castigo y tiempo de reflexión tras recompensa). En el primer conjunto de análisis se incluyeron las puntuaciones totales en el PPI-R como factor continuo entre-sujetos. En el segundo conjunto de análisis, se incluyeron las puntuaciones por factores del PPI-R (PPI-R-I, «Dominancia/Ausencia de Miedo», y PPI-R-II, «Impulsividad Egocéntrica») como factores continuos entre-sujetos. En el tercer conjunto de análisis se incluyeron las puntuaciones de las ocho subescalas del PPI-R como factores continuos entre-sujetos. La edad de los participantes fue incluida como covariante en todos los conjuntos de análisis. Este tipo de análisis permite establecer los mejores predictores de la variable dependiente de interés, dado que el poder predictivo de cada variable independiente sobre la dependiente se pone a prueba manteniendo constantes los demás predictores, lo que posibilita determinar la varianza única que explica cada factor de forma independiente. Los valores de eta al cuadrado parcial $(\eta 2 p)$ se presentan como índices del tamaño de los efectos observados.

\section{Resultados}

Los resultados del primer MLG no mostraron efectos significativos de las puntuaciones totales en el PPI-R sobre el número de cartas jugadas $(F<1)$, las ganancias totales obtenidas $(F<1)$ o el tiempo de reflexión tras recompensa $(p=.21)$. Sí se observó una tendencia a que las puntuaciones totales en PPI-R estuvieran relacionadas inversamente con el tiempo de reflexión tras castigo $\left(F[1,61]=3.75 ; p=.058 ; t=-1.94 ; \eta_{p}{ }^{2}=.06\right)$.

En el segundo MLG, no se encontraron efectos significativos de las puntuaciones del PPI-I sobre ninguna variable dependiente (número de cartas jugadas, tiempo de reflexión tras castigo o el tiempo de reflexión tras recompensa, $F s<1$; ganancias totales obtenidas, $p=.27$ ), ni tampoco del PPI-II (número de cartas jugadas, ganancias totales obtenidas o tiempo de reflexión tras recompensa, $F s<1$; tiempo de reflexión tras castigo, $p=.08$ ).

Los resultados del tercer MLG mostraron que únicamente la subescala de Ausencia Despreocupada de Planes ( $\mathrm{CN}$ ) mostraba una relación estadísticamente significativa con las cuatro variables dependientes de la CPT (como se puede observar en las tablas 1, 2, 3 y 4).

Así, las puntuaciones en $\mathrm{CN}$ estaban relacionadas significativamente de forma directa con el número de cartas jugadas -explicando de forma única un $11.6 \%$ de la varianza (véase la tabla 1)-, y de forma inversa con las ganancias totales obtenidas $(8.1 \%$ de la varianza explicada; véase la tabla 2 ), y los tiempos de reacción tras castigo (10.8\% de la varianza explicada; véase la tabla 3 ) y tras recompensa (6.8 \% de la varianza explicada; véase la tabla 4$)$. El resto de subescalas del PPI-R no mostró relaciones significativas con ninguna de las variables dependientes, salvo la subescala de Influencia Social (SOI) con el tiempo de reflexión tras recompensa (6.8 \% de la varianza). 
Tabla 1

Resultados del tercer MLG para el Número de Cartas Jugadas

\begin{tabular}{lcccc}
\hline VARIABLES INDEPENDIENTES & $F$ & $P$ & $T$ & H2P \\
\hline Edad & 1.72 & .196 & 1.31 & .032 \\
Frialdad Emocional (C) & .198 & .658 & -.445 & .004 \\
Inmunidad al Estrés (STI) & .325 & .571 & .570 & .006 \\
Ausencia de Miedo (F) & .021 & .884 & -.147 & .000 \\
Influencia Social (SOI) & 1.18 & .283 & 1.09 & .022 \\
Egocentrismo Maquiavélico (ME) & .114 & .738 & -.337 & .002 \\
Inconformismo Rebelde (RN) & .315 & .577 & -.561 & .006 \\
Externalización de la Culpa (BE) & 2.5 & .120 & -1.58 & .046 \\
Ausencia Despreocupada de Planes (CN) & 6.80 & .012 & 2.61 & .116 \\
\hline
\end{tabular}

Nota. Los valores en negrita son significativos a un nivel de $p<.05$.

Tabla 2

Resultados del tercer MLG para las Ganancias totales obtenidas

\begin{tabular}{lcccc}
\hline VARIABLES INDEPENDIENTES & $F$ & $P$ & $\mathrm{~T}$ & $\mathrm{H} 2 \mathrm{P}$ \\
\hline Edad & .701 & .406 & -.837 & .013 \\
Frialdad Emocional (C) & .124 & .727 & .351 & .002 \\
Inmunidad al Estrés (STI) & .871 & .355 & -.933 & .016 \\
Ausencia de Miedo (F) & .000 & .996 & .005 & .000 \\
Influencia Social (SOI) & .084 & .363 & -.919 & .016 \\
Egocentrismo Maquiavélico (ME) & .589 & .446 & .768 & .011 \\
Inconformismo Rebelde (RN) & .195 & .660 & .442 & .004 \\
Externalización de la Culpa (BE) & .647 & .425 & .804 & .012 \\
Ausencia Despreocupada de Planes (CN) & 4.61 & .037 & -2.15 & .081 \\
\hline
\end{tabular}

Nota. Los valores en negrita son significativos a un nivel de $p<.05$. 
Tabla 3

Resultados del tercer MLG para el Tiempo de Reflexión tras Castigo

\begin{tabular}{lcccc}
\hline VARIABLES INDEPENDIENTES & $F$ & $P$ & $T$ & H2P \\
\hline Edad & .769 & .384 & .877 & .015 \\
Frialdad Emocional (C) & .003 & .958 & -.053 & .000 \\
Inmunidad al Estrés (STI) & .460 & .501 & -.678 & .009 \\
Ausencia de Miedo (F) & 1.03 & .314 & 1.02 & .019 \\
Influencia Social (SOI) & 2.21 & .143 & -1.49 & .041 \\
Egocentrismo Maquiavélico (ME) & .008 & .928 & -.091 & .000 \\
Inconformismo Rebelde (RN) & .173 & .679 & -.416 & .003 \\
Externalización de la Culpa (BE) & .085 & .772 & -.291 & .002 \\
Ausencia Despreocupada de Planes (CN) & 6.30 & .015 & -2.51 & .108 \\
\hline
\end{tabular}

Nota. Los valores en negrita son significativos a un nivel de $p<.05$.

Tabla 4

Resultados del tercer MLG para el Tiempo de Reflexión tras Recompensa

\begin{tabular}{lcccc}
\hline VARIABLES INDEPENDIENTES & $F$ & $P$ & $\mathrm{~T}$ & $\mathrm{H} 2 \mathrm{P}$ \\
\hline Edad & .112 & .739 & .335 & .002 \\
Frialdad Emocional (C) & .084 & .773 & .290 & .002 \\
Inmunidad al Estrés (STI) & .000 & .989 & .014 & .000 \\
Ausencia de Miedo (F) & .480 & .492 & .693 & .009 \\
Influencia Social (SOI) & 3.78 & .057 & -1.96 & .068 \\
Egocentrismo Maquiavélico (ME) & .344 & .560 & -.587 & .007 \\
Inconformismo Rebelde (RN) & .132 & .718 & .363 & .003 \\
Externalización de la Culpa (BE) & .126 & .724 & .355 & .002 \\
Ausencia Despreocupada de Planes (CN) & 3.81 & .056 & -1.95 & .068 \\
\hline
\end{tabular}




\section{Discusión y conclusiones}

El objetivo del presente estudio era examinar la existencia de una perseveración de respuestas no adaptativas asociada a los rasgos psicopáticos en una muestra no clínica de estudiantes universitarios. Los resultados obtenidos mostraron que la subescala de Ausencia Despreocupada de Planes del Psychopathic Personality Inventory-Revised-que refleja características de impulsividad, dificultades en aprender de los propios errores y falta de planificación de la psicopatía (Lilienfeld y Widows, 2005)- predecía, de forma exclusiva, un mayor número de cartas jugadas y un menor número de ganancias totales, así como una falta de reflexión general tras el feedback (sea de recompensa o de castigo). Este resultado es consistente con la literatura previa que indica que los déficits en la perseveración de respuestas encontrados en psicópatas encarcelados están específicamente relacionados con las características de un estilo de vida impulsivo e irresponsable de la psicopatía (Moltó et al., 2007) y que, lejos de ser un déficit exclusivo de dicha condición patológica, parece estar presente en otros grupos psicopatológicos como el trastorno disocial (Fonseca y Yule, 1995), niños con trastorno disocial y TDAH (Matthys et al., 1998), niños agresivos (Séguin et al., 2002), adultos jóvenes con trastorno disocial e historia de trastorno por hiperactividad (Fisher et al., 2005), jugadores patológicos (Goudriaan et al., 2005) o asociado al uso/abuso de sustancias (Martin et al., 2000), condiciones todas ellas englobadas dentro del espectro de los trastornos externalizantes (Krueger et al., 2002, 2007).

Por otro lado, existen muy pocos estudios que hayan examinado el papel que juega la conducta reflexiva en la perseveración de respuestas no adaptativas. De manera consistente con estudios previos llevados a cabo con psicópatas encarcelados (Moltó et al., 2007), nuestros resultados mostraron que las características de la psicopatía relacionadas con una peor ejecución en la CPT se asociaron también a menores tiempos de reflexión tras castigo y tras recompensa. Este resultado parece no dar soporte empírico a la interpretación propuesta por Newman et al. (1987) que relaciona una peor ejecución en esta tarea con una falta de reflexión tras los castigos. Así, más que una falta de procesamiento de información de las señales de castigo, la peor ejecución en la CPT se encuentra asociada de forma más clara con las características de impulsividad, falta de planificación y necesidad de estimulación de la psicopatía, probablemente debido a que la necesidad de actuar y responder de estas personas dificulta el procesamiento de señales tanto de recompensa como de castigo, lo que a su vez reduce su sensibilidad a los cambios en las contingencias de la tarea.

Aunque en la presente muestra subclínica las puntuaciones totales en el PPI-R no se asociaran con una perseveración de respuestas no adaptativas -como sí se ha demostrado en los estudios previos utilizando el PCL-R en muestras penitenciarias (Newman et al., 1987; Moltó et al., 2007)-, los resultados del presente trabajo son consistentes con la propuesta de que el diagnóstico de psicopatía del PPI-R, más que representar un constructo unitario (como es la evaluación de la psicopatía a partir del PCL-R), constituye un rasgo multifacético que emerge a partir de rasgos de orden menor, que pueden no estar relacionados entre sí o mostrar relaciones opuestas (Lilienfeld y Widows, 2005). El hecho de que la escala de Ausencia Despreocupada de Planes, tras controlar la varianza compartida con el resto de subescalas, se asociara con una perseveración de respuestas no adaptativas en la CPT y con un estilo de juego caracterizado por una falta de reflexión general tras el feedback, constituye un soporte empírico a la medida que ofrece dicha subescala, ya que precisamente refleja las características de impulsividad que tradicionalmente se han asociado a la psicopatía.

Como se ha comentado a lo largo del presente trabajo, existe un importante cuerpo de evidencia empírica que relaciona la perseveración de respuestas no adaptativas y diversos grupos psicopatológicos relacionados con el factor general de vulnerabilidad externalizante. 
Pues bien, los resultados del presente estudio son consistentes con los modelos duales del trastorno que entienden la psicopatía como un constructo dual (Fowles y Dindo, 2006, 2009; Patrick, 2007; Patrick y Bernat, 2009). Diversos estudios de laboratorio han puesto de manifiesto que los dos grandes factores del PPI-R (PPI-I, que refleja parte de las características afectivo-interpersonales y al que parece subyacer una disposición temperamental de bajo miedo, y PPI-II, que refleja las características de desviación social, al que subyacería una disposición de vulnerabilidad a problemas externalizantes) se asocian a distintos déficits psicofisiológicos y conductuales. Así, existe evidencia de un déficit en la activación del sistema motivacional defensivo asociado a las puntuaciones en el PPI-I (Dominancia/Ausencia de Miedo), pero no al PPI-II, medido a través de distintas tareas de laboratorio, como una menor potenciación en el reflejo de sobresalto (Benning, Patrick y lacono, 2005), una reducción en el segundo componente acelerativo de la respuesta cardiaca de defensa (Esteller et al., 2010) o un déficit en el condicionamiento clásico del miedo (López, Poy, Patrick y Moltó, 2013). Por su parte, el PPI-Il se ha asociado de manera específica con una hipersensibilidad del sistema dopaminérgico mesencefálico -lo que sugiere una mayor sensibilidad a la recompensa (Buckholtz et al., 2010)y con una reducida amplitud del componente P300 -lo que podría reflejar déficits en la distribución de la atención hacia estímulos relevantes durante la ejecución de tareas cognitivas (Carlson, Thái y McLarnon, 2009). A nivel conductual se han encontrado déficits en la inhibición de respuestas en baterías de pruebas neuropsicológicas asociados a las puntuaciones en el PPI-II, explicados particularmente por las subescalas de Ausencia Despreocupada de Planes y Maquiavelismo Egocéntrico (Sellbom y Verona, 2007).

En este sentido, la asociación específica entre la subescala de Ausencia Despreocupada de Planes (que forma parte del PPI-II) y la perseveración de respuestas no adaptativas parece respaldar esta conceptuación de la psicopatía, de forma paralela a los resultados obtenidos en psicópatas encarcelados (Moltó et al., 2007).

En resumen, el constructo de psicopatía parece no ser de naturaleza unitaria, sino que representa una constelación de múltiples rasgos que pueden dar lugar a diferentes manifestaciones fenotípicas (Skeem et al., 2011). Los resultados del presente estudio subrayan la importancia de diferenciar entre los diferentes componentes relacionados con el constructo a la hora de asociarlos a distintos déficits de laboratorio, que puedan servir como marcadores de las distintas dimensiones del trastorno, ayudando así a validar las distintas escalas y dimensiones de los instrumentos diseñados para la evaluación de la psicopatía en población no penitenciaria.

Finalmente, cabe hacer referencia a las limitaciones del presente estudio. Por un lado, la muestra utilizada estaba compuesta únicamente por estudiantes universitarios, por lo que algunas variables, como el alto nivel educativo, quizá hayan podido influir en los resultados limitando por este motivo la posibilidad de generalizar las conclusiones al concepto de psicopatía en la población general. Futuros estudios con muestras más amplias que puedan cubrir el rango de dimensiones asociadas al constructo parecen necesarios con el fin de caracterizar mejor la naturaleza y los correlatos del trastorno. Por otro lado, como nuestro estudio se centró en una muestra de varones - dada la falta de evidencia concluyente acerca de la perseveración de respuestas no adaptativas en mujeres psicópatas institucionalizadas (cf. Vitale y Newman, 2001) - no parece justificada la formulación de hipótesis claras respecto a lo que cabe esperar en poblaciones no penitenciarias sobre la relación entre la perseveración de respuestas no adaptativas y la psicopatía en mujeres. En este sentido, futuros estudios podrían atender a las diferencias de género para ayudar a entender las similitudes y diferencias entre las manifestaciones del constructo en mujeres y hombres. 


\section{Referencias bibliográficas}

Benning, S. D., Patrick, C. J., Hicks, B. M., Blonigen, D. M. \& Krueger, R. F. (2003). Factor structure of the Psychopathic Personality Inventory: Validity and implications for clinical assessment. Psychological Assessment, 15(3), 340-350.

Benning, S. D, Patrick, C. J. \& lacono, W. (2005). Psychopathy, startle blink modulation, and electrodermal reactivity in twin men. Psychophysiology, 42, 753-762.

Buckholtz, J. W., Treadway, M. T., Cowan, R. L., Woodward, N. D., Benning, S. D., Li, R. et al. (2010). Mesolimbic dopamine reward system hypersensitivity in individuals with psychopathic traits. Nature Neuroscience, 13, 419-421.

Carlson, S. R., Thái, S. \& Mclarnon, M. E. (2009). Visual P3 amplitude and self reported psychopathic personality traits: Frontal reduction is associated with self-centered impulsivity. Psychophysiology, 46, 100-113.

Cleckley, H. (1941/1976). The mask of sanity. St. Louis, MO: Mosby.

Esteller, A., Segarra, P., Fonfría, A., Poy, R., López, R., Pastor, M. C., Molés, M. \& Moltó, J. (2010). Cardiac defense response and subclinical pyschopathy [Abstract]. Psychophysiology, 47, S41.

Fischer, M., Barkley, R., Smallish, L. \& Fletcher, K. (2005). Executive functioning in hyperactive children as young adults: Attention, inhibition, response perseveration, and the impact of comorbidity. Developmental Neuropsychology, 27, 107-133.

Fisher, L. \& Blair, R. J. R. (1998). Cognitive impairment and its relationship to psychopathic tendencies in children with emotional and behavioral difficulties. Journal of Abnormal Child Psychology, 26, 511-519.

Fonseca, A. C. \& Yule, W. (1995). Personality and antisocial behavior in children and adolescents: An enquiry into Eysenck's and Gray's theories. Journal of Abnormal Child Psychology, 23, 767-781.

Fowles, D. C. \& Dindo, L. (2006). A dual-deficit model of psychopathy. En C. J. Patrick (ed.), Handbook of Psychopathy (pp. 14-34). New York: Guilford Press.

Fowles, D. C. \& Dindo, L. (2009). Temperament and psychopathy: A dual-pathway model. Current Directions in Psychological Science, 18, 179-183.

Goudriaan, A., Oosterlaan, J., De Beurs, E. \& Van den Brink, W. (2005). Decision making in pathological gambling: A comparison between pathological gamblers, alcohol dependents, persons with Tourette syndrome, and normal controls. Cognitive Brain Research, 23, 137-151.

Hare, R. D. (1991). Hare Psychopathy Checklist-Revised. Toronto, ON: Multi-Health Systems.

Hare, R. D. (2003). Hare Psychopathy Checklist-Revised (2. ${ }^{a}$ ed.). Toronto, ON: Multi-Health Systems.

Krueger, R. F., Hicks, B. M., Patrick, C. J., Carlson, S. R., lacono, W. G. \& McGue, M. (2002). Etiologic connections among substance dependence, antisocial behavior, and personality: Modeling the externalizing spectrum. Journal of Abnormal Psychology, 111, 411-424.

Krueger, R. F., Markon, K. E., Patrick, C. J., Benning, S. D. \& Kramer, M. D. (2007). Linking antisocial behavior, substance use, and personality: An integrative quantitative model of the adult externalizing spectrum. Journal of Abnormal Psychology, 116, 645-666.

Lilienfeld, S. O. \& Widows, M. R. (2005). Psychopathic Personality Inventory-Revised (PPI-R) professional manual. Odessa, FL: Psychological Assessmen Resources.

López, R. (2010). Déficit en el sistema motivacional aversivo en psicópatas subclínicos evaluados mediante el Psychopathic Personality Inventory-Revised (PPI-R). Tesis doctoral. Universitat Jaume I, Castellón, España Obtenido de: https://www.educacion.gob.es/teseo/imprimirFicheroTesis.do?fichero $=15603$ 
López, R., Poy, R., Patrick, C. J. \& Moltó, J. (2013). Deficient fear conditioning and self-reported psychopathy: The role of fearless dominance. Psychophysiology, 50, 210-218.

Martin, C. A., Rayens, M. K., Kelly, T., Hartung, C. \& Leukefeld, C. (2000). Card perseveration task performance and post-task feeling states: Relationship to drug use in adolescents. American Journal of Drug and Alcohol Abuse, 26, 325-333.

Matthys, W., Van Goozen, S. H., De Vries, H., Cohen- Kettenis, P. T. \& Van Engeland, H. (1998). The dominance of behavioural activation over behavioural inhibition in conduct disordered boys with or without attention deficit hyperactivity disorder. Journal of Child Psychology and Psychiatry, 39, 643-653.

Matthys, W., Van Goozen, S. H., Snoek, H. \& Van Engeland, H. (2004). Response perseveration and sensitivity to reward and punishment in boys with oppositional defiant disorder. European Child and Adolescent Psychiatry, 13, 362-364.

McCleary, R. A. (1996). Response modulating function of the limbic system: Initiation and suppression. En E. Stellar y J. M. Sprague (eds.), Progress in physiological psychology (Vol. 1, pp. 209-271). San Diego, CA: Academic Press.

Moltó, J., Poy, R., Segarra, P., Pastor, M. C. \& Montañés, S. (2007). Response perseveration in psychopaths: Interpersonal/Affective or Social deviance traits? Journal of Abnormal Psychology, 116, 632-637.

Newman, J. P., Patterson, C. M. \& Kosson, D. S. (1987). Response perseveration in psychopaths. Journal of Abnormal Psychology, 96(2), 145-148.

Newman, J. P. \& Wallace, J. F. (1993). Diverse pathways to deficient self-regulation: implications for disinhibitory psychopathology in children. Clinical Psychology Review, 15, 699-720.

Patrick, C. J. (2007). Getting to the heart of psychopathy. In H. Hervé y J. Yuille (eds.), The psychopath: Theory, research, and practice (pp. 207-252). Mahwah, NJ: Erlbaum.

Patrick, C. J. \& Bernat, E. M. (2009). Neurobiology of psychopathy: A two-process theory. En G. G. Berntson \& Cacioppo, J. T. (eds.), Handbook of neuroscience for the behavioral sciences (pp. 1110-1131). New York: John Wiley \& Sons.

Patrick, C. J., Hicks, B. M., Krueger, R. F. \& Lang, A. R. (2005). Relations between psychopathy facets and externalizing in a criminal offender sample. Journal of Personality Disorders, 19 , 339-356.

Patterson, C. M. \& Newman, J. P. (1993). Reflectivity and learning from aversive events: Toward a psychological mechanism for the syndromes of disinhibition. Psychological Review, 100, 716-736.

Séguin, J. R., Arseneault, L., Boulerice, B., Harden, P. W. \& Tremblay, R. E. (2002). Response perseveration in adolescent boys with stable and unstable histories of physical aggression: The role of underlying processes. Journal of Child Psychology and Psychiatry, 43, 481-494.

Sellbom, M. \& Verona, E. (2007). Neuropsychological correlates of psychopathic traits in a nonincarcerated sample. Journal of Research in Personality, 41, 276-294.

Shapiro, S. K., Quay, H. C., Hogan, A. E. \& Schwartz, K. P. (1988). Response perseveration and delayed responding in undersocialized aggressive conduct disorder. Journal of Abnormal Psychology, 97, 371-373.

Siegel, R. A. (1978). Probability of punishment and suppression of behavior in psychopathic and nonpsychopathic offenders. Journal of Abnormal Psychology, 87, 514-522.

Skeem, J. L., Polaschek, D., Patrick, C. J. \& Lilienfeld, S. O. (2011). Psychopathic personality: Bridging the gap between empirical evidence and public policy. Psychological Science in the Public Interest, 12, 95-162.

Vitale, J. E. \& Newman, J. P. (2001) Response perseveration in psychopathic women. Journal of Abnormal Psychology, 110, 644-647. 
White, J. L., Moffitt, T. E., Caspi, A., Bartusch, D. J., Needles, D. J. \& Stouthamer-Loeber, M. (1994). Measuring impulsivity and examining its relationship to delinquency. Journal of Abnormal Psychology, 103, 192-205. 